\title{
Numerical Predictions of Cavitating Flow around Model Scale Propellers by CFD and Advanced Model Calibration
}

\author{
Mitja Morgut and Enrico Nobile \\ Dipartimento di Ingegneria e Architettura, Università degli Studi Trieste, P.le Europa 1, 34127 Trieste, Italy \\ Correspondence should be addressed to Mitja Morgut, mmorgut@units.it
}

Received 3 February 2012; Accepted 5 June 2012

Academic Editor: Jules W. Lindau

Copyright ( $) 2012$ M. Morgut and E. Nobile. This is an open access article distributed under the Creative Commons Attribution License, which permits unrestricted use, distribution, and reproduction in any medium, provided the original work is properly cited.

\begin{abstract}
The numerical predictions of the cavitating flow around two model scale propellers in uniform inflow are presented and discussed. The simulations are carried out using a commercial CFD solver. The homogeneous model is used and the influence of three widespread mass transfer models, on the accuracy of the numerical predictions, is evaluated. The mass transfer models in question share the common feature of employing empirical coefficients to adjust mass transfer rate from water to vapour and back, which can affect the stability and accuracy of the predictions. Thus, for a fair and congruent comparison, the empirical coefficients of the different mass transfer models are first properly calibrated using an optimization strategy. The numerical results obtained, with the three different calibrated mass transfer models, are very similar to each other for two selected model scale propellers. Nevertheless, a tendency to overestimate the cavity extension is observed, and consequently the thrust, in the most severe operational conditions, is not properly predicted.
\end{abstract}

\section{Introduction}

In the field of marine applications, and in the particular case of marine propellers, the onset of cavitation is, in general, associated with negative design implications such as thrust reduction, noise, vibration, and erosion. In the last decades, also owing to the steady increasing of the computer performances, in order to improve the design process, several CFD (Computational Fluid Dynamics) methods have been developed for the prediction of the cavitation appearance and the estimation of its effects.

In this study, we evaluated the capabilities of the homogeneous, that is, one-fluid model implemented in the ANSYS CFX 12 (for brevity CFX hereafter) commercial CFD solver, for the prediction of cavitating flow around model scale propellers working in uniform inflow. This model treats the cavitating flow as a mixture of two fluids behaving as a single one. The set of the governing equations is composed by the (volume) continuity and momentum equations for the mixture, plus a transport equation for the water volume fraction. The mass transfer rate due to cavitation is regulated by the same source term appearing in the (volume) continuity and volume fraction equations. This source term, in CFX, using the default setting is modelled by employing the mass transfer model originally proposed by Zwart et al. [1] (Zwart for brevity). However, in literature several other mass transfer models are available (see [2]). Thus, in order to improve the reliability of the simulations besides the Zwart model we employed also two other widespread mass transfer models: the model originally proposed by Kunz et al. [3] (for brevity Kunz) and the model originally proposed by Singhal et al. [4] also known as Full Cavitation Model (FCM hereafter). The Kunz and FCM models were added to the CFX solver using CEL (CFX Expression Language).

It is fundamental to clarify that the considered mass transfer models share the common feature of employing empirical coefficients to adjust the mass transfer rate due to condensation and evaporation processes, and their values can significantly affect both the stability and accuracy of the numerical predictions. Thus, in order to correctly evaluate the influence of the three different mass transfer models on the stability and accuracy of the numerical predictions, their empirical coefficients were first properly and congruently calibrated using an optimization strategy [5]. The models 
were calibrated considering the two-dimensional stable sheet cavity flow around a NACA-66(MOD) hydrofoil. The calibrated mass transfer models were then used to investigate the cavitating flow around two model scale propellers recognized as international benchmarks, that is, E779A propeller and PPTC propeller.

The numerical results were compared with the available experimental data. For both propellers, three selected operational conditions, corresponding to the appearance of partial and/or tip vortex cavitation, were considered $[6,7]$. For such conditions, the numerical results obtained with the different calibrated mass transfer models were very similar to each other and compared well with the experimental data, even though the numerical cavitation patterns were slightly overestimated. However, in the case of E779A propeller where also the more severe operational conditions were simulated, the thrust breakdown was not properly predicted.

In the following, the homogeneous (one-fluid) model used in this study is presented first, followed by the description of the optimization strategy used to calibrate the different mass transfer models. Then the results obtained considering the cavitating flow around the two different model scale propellers are provided. Eventually, some concluding remarks are given.

\section{Mathematical Model}

Cavitating flows can be modelled using several methods. An excellent review of different methods is provided for instance by Koop [8]. In this work, we used the homogeneous transport equation-based model $[9,10]$, described in the following.

2.1. Governing Equations. As already indicated the cavitating flow is modelled as a mixture of two species, that is, vapour and liquid behaving as a single one, where both phases share the same velocity as well as pressure fields.

In the case of the RANS (Reynolds-averaged Navier Stokes) approach to turbulence, employing the eddy viscosity models and assuming both liquid and vapour phases incompressible, turbulent cavitating flows can be described by the following set of governing equations:

$$
\begin{gathered}
\nabla \cdot \mathbf{U}=\dot{m}\left(\frac{1}{\rho_{L}}-\frac{1}{\rho_{V}}\right), \\
\frac{\partial(\rho \mathbf{U})}{\partial t}+\nabla \cdot(\rho \mathbf{U} \mathbf{U})=-\nabla P+\nabla \\
\cdot\left[\left(\mu+\mu_{t}\right)\left(\nabla \mathbf{U}+(\nabla \mathbf{U})^{T}\right)\right]+S, \\
\frac{\partial \gamma}{\partial t}+\nabla \cdot(\gamma \mathbf{U})=\frac{\dot{m}}{\rho_{L}},
\end{gathered}
$$

which are, in order, the volume continuity and the momentum equation for the liquid-vapour mixture and the volume fraction equation for the liquid phase. In the above equations, $\rho_{L}\left(\mathrm{~kg} / \mathrm{m}^{3}\right)$ and $\rho_{V}\left(\mathrm{~kg} / \mathrm{m}^{3}\right)$ are the liquid and vapour densities, respectively. $\mathbf{U}(\mathrm{m} / \mathrm{s})$ is the averaged velocity and $P \quad(\mathrm{~Pa})$ the averaged pressure. $S$ represents the additional momentum sources (e.g., the Coriolis and centrifugal forces in the rotating frame of reference). $\gamma$ is the water volume fraction which is related to the vapour volume fraction $\alpha$ through the volume fraction constraint:

$$
\gamma+\alpha=1
$$

The mixture density $\rho\left(\mathrm{kg} / \mathrm{m}^{3}\right)$ and laminar dynamic viscosity $\mu(\mathrm{kg} / \mathrm{ms})$ are evaluated according to

$$
\begin{aligned}
& \rho=\gamma \rho_{L}+(1-\gamma) \rho_{V}, \\
& \mu=\gamma \mu_{L}+(1-\gamma) \mu_{V},
\end{aligned}
$$

where $\mu_{L}(\mathrm{~kg} / \mathrm{ms})$ and $\mu_{V}(\mathrm{~kg} / \mathrm{ms})$ are the liquid and vapour dynamic viscosities, respectively. $\dot{m}\left(\mathrm{~kg} / \mathrm{m}^{3} \mathrm{~s}\right)$ represents the interphase transfer rate due to cavitation. In this study, $\dot{m}$ was modelled using alternatively the three different mass transfer models presented in the next section.

Finally, in order to close the system of the governing equations, the mixture turbulent viscosity $\mu_{t}(\mathrm{~kg} / \mathrm{ms})$ was evaluated using the eddy viscosity turbulence models developed for the single phase flow. In particular, the twoequations standard $k-\varepsilon$ and SST (shear stress transport) turbulence models were employed. For the description of the above turbulence models we refer to [10-12].

2.2. Mass Transfer Models. The mass transfer models describe/regulate the interphase mass transfer rate due to cavitation. In the last two decades, several authors proposed different mass transfer models.

In the following, we provide a brief description of three different mass transfer models employed in this study, where the interphase mass transfer rate due to cavitation was assumed positive if directed from vapour to water. These models, except for the full cavitation model, are simplified versions of the original formulations, where the contribution of the dissolved gasses was omitted.

2.2.1. Zwart Model. The Zwart model is the native CFX mass transfer model. It is based on the simplified Rayleigh-Plesset equation for bubble dynamics [13]:

$$
\dot{m}= \begin{cases}-F_{e} \frac{3 r_{\mathrm{nuc}}(1-\alpha) \rho_{V}}{R_{B}} \sqrt{\frac{2}{3} \frac{P_{V}-P}{\rho_{L}}} & \text { if } P<P_{V} \\ F_{c} \frac{3 \alpha \rho_{V}}{R_{B}} \sqrt{\frac{2}{3} \frac{P-P_{V}}{\rho_{L}}} & \text { if } P>P_{V} .\end{cases}
$$

In the above equations, $P_{V}$ is the vapour pressure, $r_{\text {nuc }}$ is the nucleation site volume fraction, $R_{B}$ is the radius of a nucleation site, and $F_{e}$ and $F_{c}$ are two empirical calibration coefficients for the evaporation and condensation processes, respectively. In CFX, the above mentioned coefficients, by default, are set as follows: $r_{\text {nuc }}=5.0 \times 10^{-4}, R_{B}=2.0 \times$ $10^{-6} \mathrm{~m}, F_{e}=50$, and $F_{c}=0.01$.

Moreover, the above equations show that expressions for condensation and evaporation are not symmetric. 
In particular, in the expression for evaporation, $\alpha$ is replaced by $r_{\text {nuc }}(1-\alpha)$ to take into account that, as the vapour volume fraction increases, the nucleation site density must decrease accordingly.

2.2.2. Full Cavitation Model. The mass transfer model proposed by Singhal et al. [4], originally known as full cavitation model, is currently employed in some commercial CFD codes, that is, FLUENT [14] and PUMPLINX [15]. This model is also based on the reduced form of the RayleighPlesset equation for bubble dynamics, and its formulation states as follows:

$$
\dot{m}= \begin{cases}-C_{e} \frac{\sqrt{k}}{T} \rho_{L} \rho_{V} \sqrt{\frac{2}{3} \frac{P_{V}-P}{\rho_{L}}}\left(1-f_{V}\right) & \text { if } P<P_{V} \\ C_{c} \frac{\sqrt{k}}{T} \rho_{L} \rho_{L} \sqrt{\frac{2}{3} \frac{P-P_{V}}{\rho_{L}}} f_{V} & \text { if } P>P_{V},\end{cases}
$$

where $f_{V}$ is the vapour mass fraction, $k\left(\mathrm{~m}^{2} / \mathrm{s}^{2}\right)$ is the turbulent kinetic energy, $T(\mathrm{~N} / \mathrm{m})$ is the surface tension, and $C_{e}=0.02$ and $C_{c}=0.01$ are two empirical calibration coefficients.

It is important to note that in this work, for convenience, we did not use the original formulation of the model, but the formulation derived by Huuva [16] in which the vapour mass fraction, $f_{V}$, is replaced by the vapour volume fraction $\alpha$.

2.2.3. Kunz Model. The Kunz mass transfer model is based on the work of Merkle et al. [17] and currently is one of the mass transfer models implemented in the OpenFOAM library [18]. In this model, unlike the above mentioned models, the mass transfer is based on two different strategies for creation $\dot{m}^{+}$and destruction $\dot{m}^{-}$of liquid. The transformation of liquid to vapour is calculated as being proportional to the amount by which the pressure is below the vapour pressure. The transformation of vapour to liquid, otherwise, is based on a third-order polynomial function of volume fraction, $\gamma$. The specific mass transfer rate is defined as $\dot{m}=$ $\dot{m}^{+}+\dot{m}^{-}$:

$$
\begin{gathered}
\dot{m}^{+}=\frac{C_{\text {prod }} \rho_{V} \gamma^{2}(1-\gamma)}{t_{\infty}}, \\
\dot{m}^{-}=\frac{C_{\text {dest }} \rho_{V} \gamma \min \left[0, P-P_{V}\right]}{\left(1 / 2 \rho_{L} U_{\infty}^{2}\right) t_{\infty}} .
\end{gathered}
$$

In the above equations, $U_{\infty}(\mathrm{m} / \mathrm{s})$ is the free-stream velocity and $t_{\infty}=L / U_{\infty}$ is the mean flow time scale, where $L$ is the characteristic length scale. $C_{\text {dest }}$ and $C_{\text {prod }}$ are two empirical coefficients. In the original formulation $C_{\text {dest }}=100, C_{\text {prod }}=$ 100.

\section{Calibration of Mass Transfer Models}

As described in the former section, in the homogeneous transport equation-based model, a particular mass transfer model which regulates the mass transfer rate from liquid to vapour and back is needed in order to simulate cavitating flow.

In this study, we employed the Zwart model, the FCM model, and the Kunz model. As already indicated in the previous section, these models employ empirical coefficients to tune the models of condensation and evaporation processes, that in turn can influence the accuracy and stability of the numerical predictions.

Thus, in order to ensure, for all three different mass transfer models, the stability and a good level of accuracy of the predictions, the empirical coefficients of the three different mass transfer models were properly calibrated using an optimization strategy. The entire calibration process was driven by the modeFRONTIER 4.2 optimization system and is described in the following. We remind that modeFRONTIER [19] is a general integration and multiobjective optimization platform, which can drive a variety of CAE packages and provide statistical and visualization tools. It is commonly used for functional and shape optimization of systems and devices, for example, see [20]. It is worth to say that with the developed strategy, the empirical coefficients were tuned considering the two-dimensional sheet cavity flow around the NACA66(MOD) hydrofoil [21]. In this manner, due to the lower computational costs, we could explore, in a reasonable computational time and with limited computational resources, a significant number of coefficients' combinations. Consequently, we had also the opportunity to verify if the coefficients calibrated for the hydrofoil case, could have a general character, and could be successfully applied to the propeller flow problem.

3.1. The Idea and Logic of the Optimization Strategy. In the developed optimization strategy, the three different mass transfer models were properly calibrated by searching the values of the empirical coefficients which minimized the objective function $f$. The objective function represented the differences between the numerical and experimental pressure distributions on the suction side of a NACA66(MOD) hydrofoil evaluated at an angle of attack $\mathrm{AoA}=4^{\circ}$ and for three different cavitating flow regimes. More precisely, the objective function $f$ was expressed as follows:

$$
f=\sum_{\sigma} \sum_{i=1}^{N}\left|C_{P_{i}}-C_{P_{i, \mathrm{Exp}}}\right| ; \quad \sigma=1.00,0.91,0.84,
$$

where $C_{P_{i}}$ and $C_{P_{i \mathrm{Exp}}}$ were the numerical and experimental values of the pressure coefficient, taken at $N=12$ locations on the suction side of the hydrofoil, and $\sigma$ was the cavitation number defined as follows:

$$
\sigma=\frac{P_{\mathrm{REF}}-P_{V}}{(1 / 2) \rho_{L} U_{\infty}^{2}}
$$

where $P_{\mathrm{REF}}(\mathrm{Pa})$ was the reference pressure and $U_{\infty}(\mathrm{m} / \mathrm{s})$ the free-stream velocity.

In order to find the values which minimized $f$, two different optimization algorithms were run in sequence. The design space was firstly explored using the MOGAII, a multiobjective genetic optimization algorithm available 
in modeFRONTIER 4.2 [19]. The MOGA-II was run for ten generations starting from an initial DOE (design of experiments) of ten designs randomly generated. In order to refine the solution, the Simplex [22], single objective optimization algorithm was used, starting from the three best solutions obtained using MOGA-II. No significant improvements were observed using the Simplex algorithm.

Figure 1 shows the logic of the optimization strategy, where the optimizer block stays for both the MOGA-II and Simplex algorithms, and where $(X 1, X 2)$ represent the couple of empirical coefficients for all three different mass transfer models.

The numerical setup adopted to run the simulations, within the optimization process, is briefly described in the following. Further details can be found in [5].

3.2. Simulation Setup. During the optimization process, the simulations were carried out using the following strategy.

The cavitating flow around the hydrofoil was simulated on the rectangular domain shown in Figure 2. The simulations were carried out in 2D and assuming steadystate conditions. For turbulence closure, the standard $k$ $\varepsilon$ turbulence model, in combination with scalable wall functions [10], was employed. The following boundary conditions were applied. On solid surfaces (top, bottom, and NACA66(MOD)), the no-slip condition was set. On outlet boundary, a fixed static pressure, $P_{\mathrm{REF}}=202,650 \mathrm{~Pa}$ was imposed and on the side faces the symmetry condition was enforced. On inlet boundary, the values of the free-stream velocity components and turbulence quantities were fixed. Water and vapour volume fractions were set equal to 1 and 0 , respectively. In order to match the experimental setup, during the numerical simulations the same Reynolds number was used. Since the water kinematic viscosity was assumed $v_{L}=8.92 \times 10^{-7} \mathrm{~m}^{2} / \mathrm{s}$, the free-stream velocity was set to $U_{\infty}=12.2(\mathrm{~m} / \mathrm{s})$. Assuming a turbulence intensity of $1 \%$, the turbulent kinetic energy $k$ and the turbulent dissipation rate $\varepsilon$ were set equal to $k=0.0223 \mathrm{~m}^{2} / \mathrm{s}^{2}, \varepsilon=0.1837 \mathrm{~m}^{2} / \mathrm{s}^{3}$ on the inlet boundary. The water density was set equal to $\rho_{L}=$ $997 \mathrm{~kg} / \mathrm{m}^{3}$ and the maximum water-vapour density ratio was limited to $\rho_{L} / \rho_{V}=1000$ in order to ensure solver stability. All the simulations were performed on a hexahedral grid with 58700 nodes which proved to give mesh independent results [5], for fully wetted flow. Figure 3 shows the computational mesh around the hydrofoil generated with the ANSYS-ICEM mesh generator (ICEM hereafter). The average value of $y^{+}$ evaluated on the solid surfaces of the hydrofoil was equal to 28. $y^{+}$was defined as $y^{+}=\mu_{\tau} y / \nu$, where $\mu_{\tau}=\left(\tau_{w} / \rho\right)^{1 / 2}$ is the friction velocity, $y$ is the normal distance from the wall, and $\tau_{w}$ is the wall shear stress.

3.3. Calibration Results. In this study, the evaporation $F_{e}$ and condensation $F_{c}$ coefficients of the Zwart model were tuned within the following ranges: $30 \leq F_{e} \leq 500,5.0 \times 10^{-4} \leq$ $F_{c} \leq 8.0 \times 10^{-2}$. The best result was found with $F_{e}=300$ and $F_{c}=0.03$.

For FCM, the values of the evaporation coefficient $C_{e}$ and of the condensation coefficient $C_{c}$ were tuned within

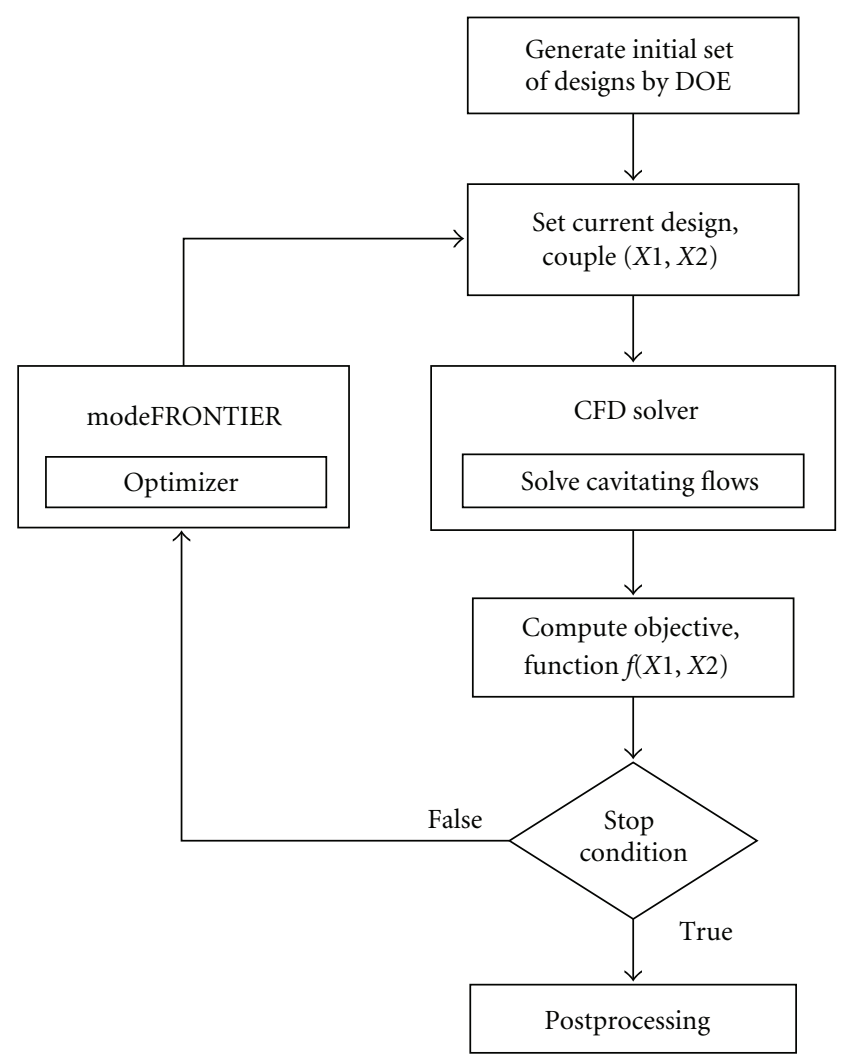

FIGURE 1: Logic of the optimization strategy.

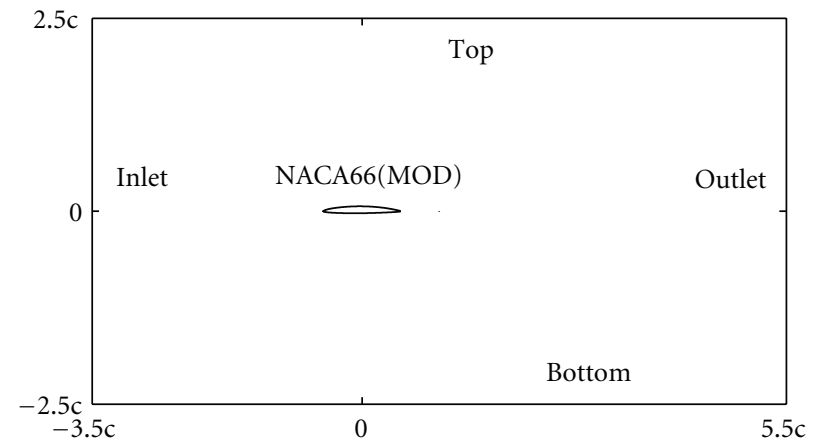

Figure 2: NACA66(MOD), shape of the computational domain.

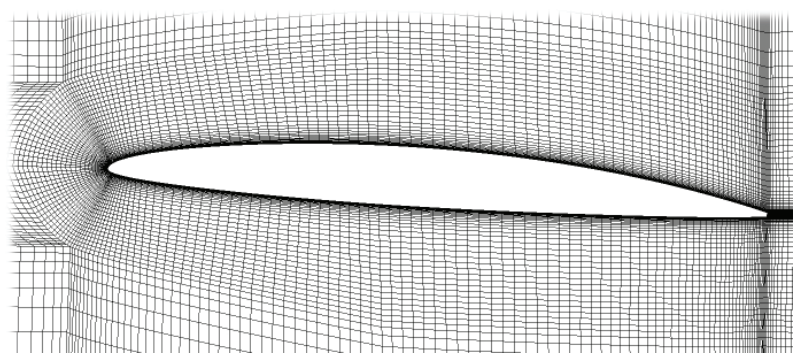

Figure 3: Mesh around the NACA66(MOD) hydrofoil. 
TABLE 1: Default and calibrated coefficients for the different mass transfer models.

\begin{tabular}{lcccccc}
\hline & \multicolumn{2}{c}{ Zwart } & \multicolumn{2}{c}{ FCM } & \multicolumn{2}{c}{ Kunz } \\
& $F_{e}$ & $F_{c}$ & $C_{e}$ & $C_{c}$ & $C_{\text {dest }}$ & $C_{\text {prod }}$ \\
\hline Default & 50 & 0.01 & 0.02 & 0.01 & 100 & 100 \\
Calibrated & 300 & 0.03 & 0.40 & $2.3 E-04$ & 4100 & 455 \\
\hline
\end{tabular}

the following ranges: $0.01 \leq C_{e} \leq 1,1.0 \times 10^{-6} \leq C_{c} \leq$ $1.0 \times 10^{-2}$. The best solution was found with $C_{e}=0.40$ and $C_{c}=2.30 \times 10^{-4}$.

For Kunz, the designs space was defined as follows: $100 \leq$ $C_{\text {dest }} \leq 5000,10 \leq C_{\text {prod }} \leq 1000$. The best solution was achieved with $C_{\text {prod }}=455$ and $C_{\text {dest }}=4100$. In the Kunz model, in order to set the mean flow time scale $t_{\infty}=L / U_{\infty}$, the chord of the hydrofoil was chosen as a length scale, $L$, following [16].

For the sake of completeness, Table 1 highlights that the empirical coefficients which resulted from the calibration process were quite different compared to the default ones. The differences between the calibrated and default values of the coefficients were lower for the Zwart model, probably because being this one the native mass transfer model of CFX, the suggested empirical values have been already found to work well for a wide range of applications.

Figure 4 shows, for instance for $\sigma=0.91$, how the cavities predicted with the noncalibrated mass transfer models had a lower vapour content and were shorter than those obtained with the calibrated models. These differences were more pronounced for the FCM and Kunz mass transfer models. As a matter of fact considering the suction side pressure distributions depicted in Figure 5, it is possible to note that with the noncalibrated mass transfer models, and especially with the Kunz and FCM mass transfer models, the numerical cavities were significantly underpredicted compared to the experimental ones, for all the three different cavitating flow regimes, that is, $\sigma=1.00,0.91$, and 0.84 . On the other hand, from the cavitation patterns presented in Figure 4, and the suction side pressure distributions given in Figure 6, it is possible to appreciate that the numerical results provided by the three different calibrated mass transfer models were very close to each other and in line with the experimental data.

Finally, let us clarify that before being applied to the predictions of the three-dimensional cavitating flow around model scale propellers, the three different calibrated mass transfer models were first assessed considering further twodimensional sheet cavity flow regimes around a hydrofoil [5].

\section{Model Scale Propellers}

4.1. Test Cases. In this study, the model scale propellers E779A and PPTC, depicted in Figure 7, were considered.

The E779A propeller is a four-bladed, fixed-pitch, and low-skew propeller, designed in 1959 with a diameter D $=0.2272 \mathrm{~m}$. Since 1997, it has been used in experimental activities performed by INSEAN (Istituto Nazionale di Studi ed Esperienze di Architettura Navale) aimed at providing
TABLE 2: Distances of the boundaries/surfaces from the propeller mid plane in axial direction for inlet, outlet, front, aft, and from the propeller rotation axis centreline in radial direction for outer and top.

\begin{tabular}{lcccccc}
\hline Propeller & Inlet & Outlet & Outer & Front & Aft & Top \\
\hline E779A & $3.40 \mathrm{D}$ & $5.35 \mathrm{D}$ & $5.00 \mathrm{D}$ & $0.40 \mathrm{D}$ & $0.35 \mathrm{D}$ & $0.70 \mathrm{D}$ \\
PPTC & $2.30 \mathrm{D}$ & $5.30 \mathrm{D}$ & $5.00 \mathrm{D}$ & $0.41 \mathrm{D}$ & $0.31 \mathrm{D}$ & $0.60 \mathrm{D}$ \\
\hline
\end{tabular}

a thorough characterization of marine propeller hydrodynamics and hydroacoustics over a wide range of operational conditions. It has been widely used for the validation of CFD codes (e.g., see [23-25]).

The PPTC (Potsdam Propeller Test Case) is a five-bladed, controllable pitch propeller having a diameter $\mathrm{D}=0.250 \mathrm{~m}$ which was used as a blind test case at the 2011 Workshop on Cavitation and Propeller Performance, [26]. A significant amount of the data covering propeller's geometry, open water tests, velocity field measurements, and cavitation tests is currently available in [27]. The experimental data were recorded in the towing tank and cavitation tunnel of the SVA (Potsdam Model Basin) [7, 28, 29].

4.2. Solution Strategy. The propellers were assumed to work in a uniform inflow and thus only one passage blade was modelled for computational convenience. Figures 8 and 9 show the shapes of the computational domains used in the case of the E779A propeller and PPTC propeller, respectively. The domains' dimensions are listed in Table 2.

Both domains were subdivided in two regions, that is, Rotating and Fixed, and the following boundary conditions were applied: on inlet boundary, the free-stream velocity components and a turbulence level of $1 \%$ were set. On outlet boundary, a fixed value of the static pressure was imposed. On the periodic boundaries (sides of the domain), the rotational periodicity was ensured. On solid surfaces the no-slip boundary condition was applied, and on outer boundary, the slip condition was set. Since the propeller rotation was simulated using the MRF (Multiple Reference Frame) approach in the stationary region called Fixed, the governing equations were solved into a fixed frame of reference, while into a rotating region called Rotating, the governing equations were solved into a rotating frame of reference. For the discretization of the advective terms, the high resolution scheme was used [10].

For turbulence closure, the two-equation SST turbulence model was used in combination with the automatic wall treatment $[10,30]$.

For a given value of the advance coefficient $J=U / n D$, a particular cavitating flow regime was set according to the cavitation number $\sigma_{n}$ defined, for the propeller case, as follows:

$$
\sigma_{n}=\frac{P_{\mathrm{REF}}-P_{V}}{0.5 \rho_{L}(n D)^{2}},
$$

where $n$ (rps) is the propeller rotational speed and $\mathrm{P}_{\mathrm{V}}(\mathrm{Pa})$ is the vapour pressure. In this study, we assumed $P_{\mathrm{REF}}=P_{\text {Outlet }}$. 

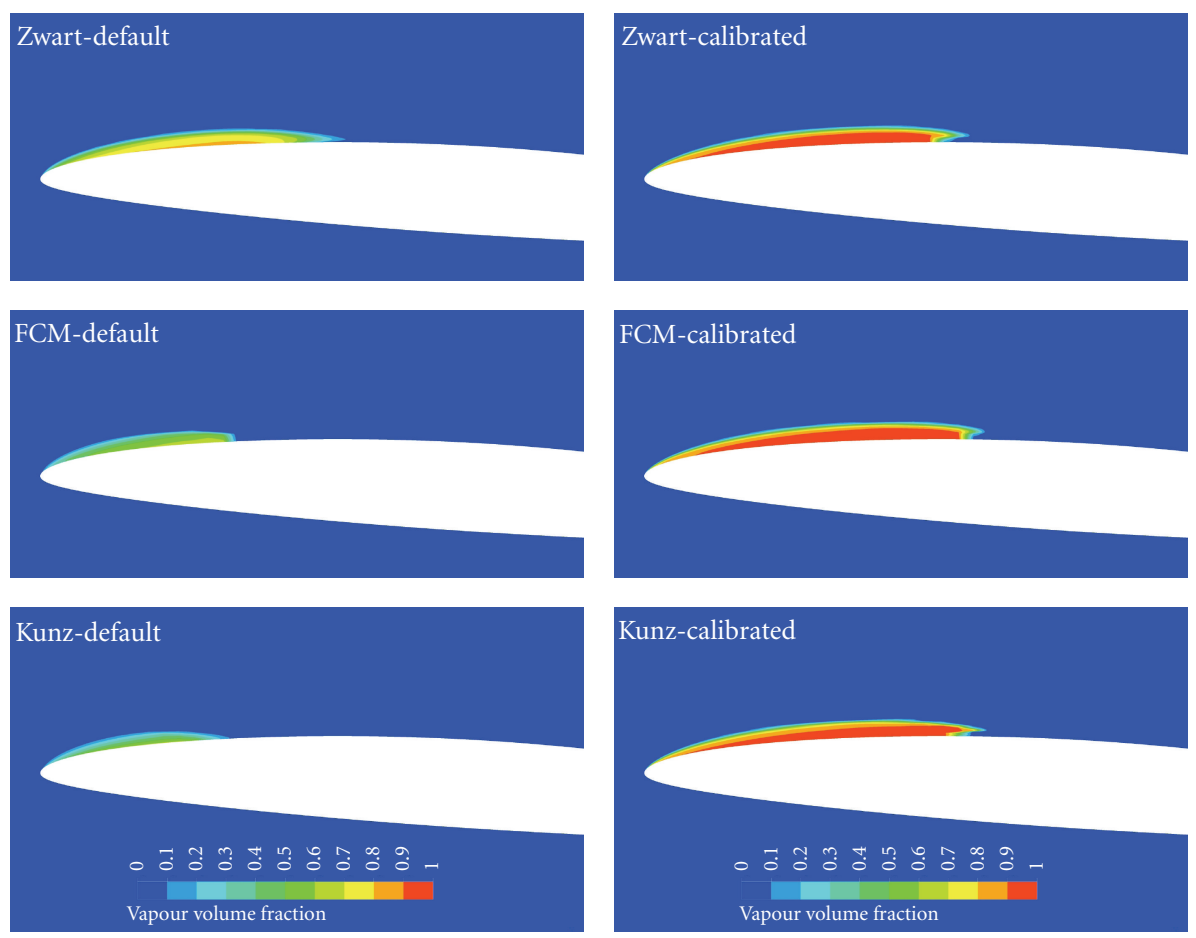

(a)

(b)

FIgURE 4: Sheet cavities over the NACA66(MOD) hydrofoil at AoA $=4^{\circ}, \operatorname{Re}=2 \times 10^{6}, \sigma=0.91$, computed using noncalibrated (a) and calibrated (b) mass transfer models.

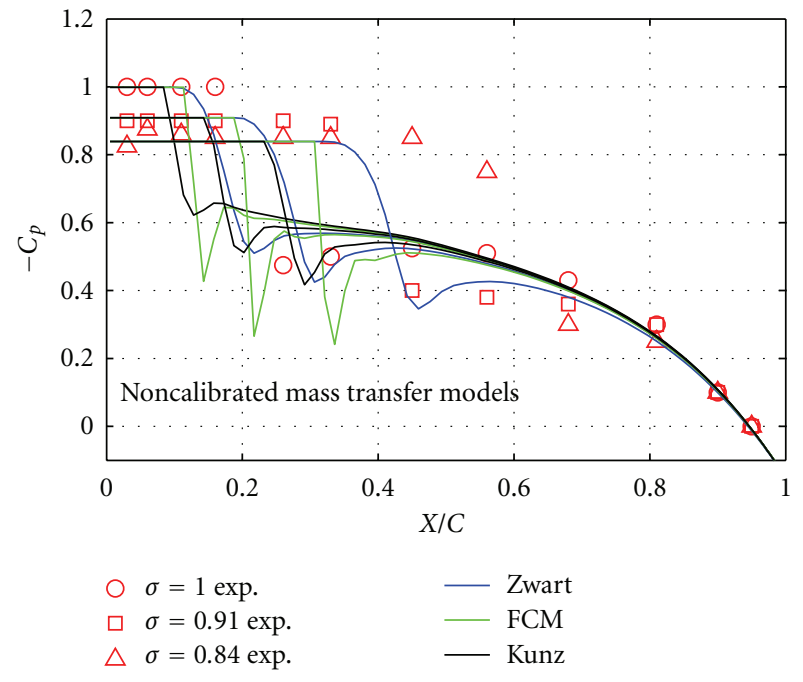

Figure 5: NACA66(MOD) hydrofoil at AoA $=4^{\circ}, \mathrm{Re}=2 \times 10^{6}$. Suction side pressure distributions computed using noncalibrated Zwart, FCM, and Kunz mass transfer models.

For all the different cavitating flow regimes, the numerical predictions were carried out using alternatively all the three different calibrated mass transfer models.

4.3. Meshing. For both propellers, the meshes of the two different domain regions, that is, Fixed and Rotating, were

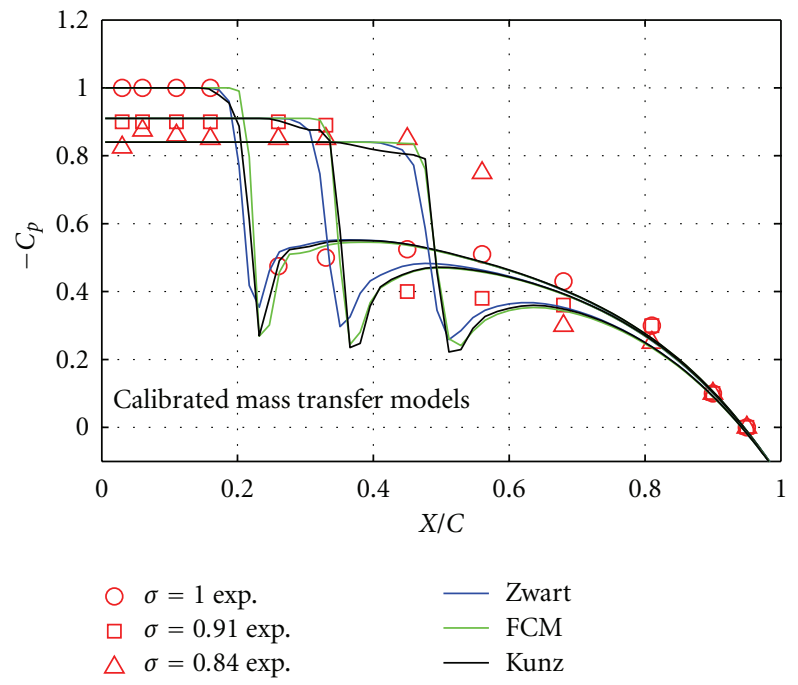

Figure 6: NACA66(MOD) hydrofoil at AoA $=4^{\circ}, \operatorname{Re}=2 \times$ $10^{6}$. Suction side pressure distributions computed using calibrated Zwart, FCM, and Kunz mass transfer models.

generated independently from each other. They were generated with ICEM without trying to ensure a 1:1 matching of the nodes at the interfaces (the common surfaces of the two different domain regions). The different domain regions were subsequently joined in CFX using the GGI (Generalized Grid Interfaces) capabilities of the solver. From Table 3, it 


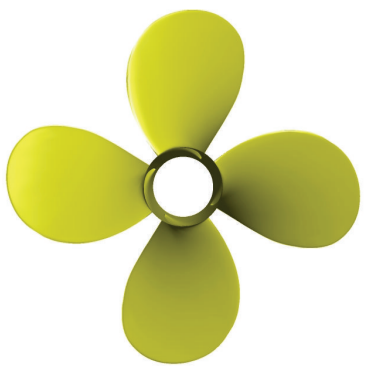

(a)

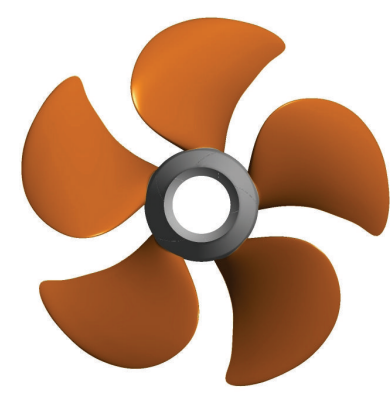

(b)

FIGURE 7: Propellers' CAD models. View looking downstream. E779A propeller (a), PPTC propeller (b). The propellers' models are not shown using the same scale.

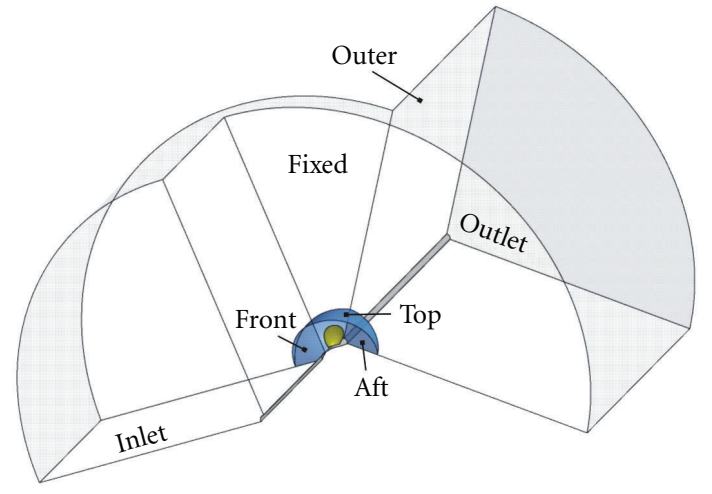

Figure 8: E779A, computational domain.

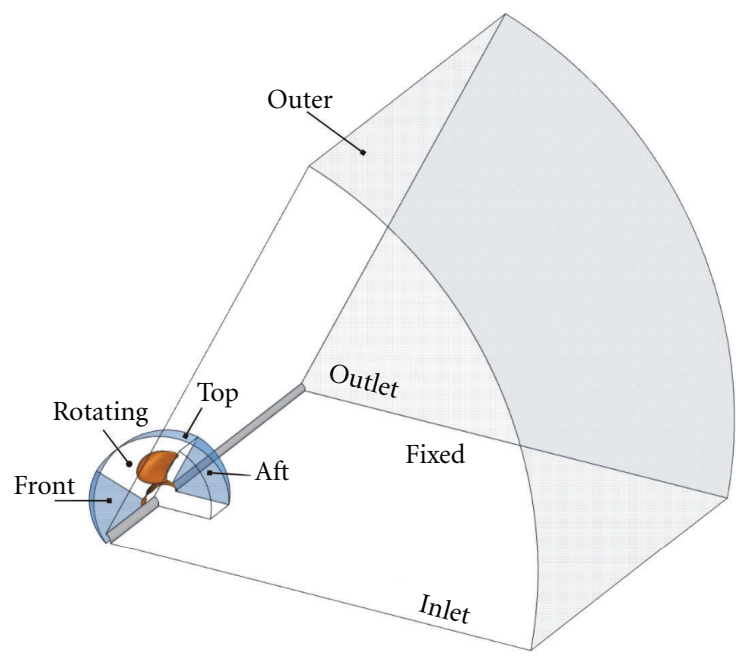

Figure 9: PPTC, computational domain.

is possible to note that for both propellers the Rotating region was discretized by a hexa-structured mesh which was generated, as suggested in [31, 32], by decomposing that domain region in a large number of blocks. The resolution and also the quality of the cells were set through a proper node distribution on the blocks edges.
TABLE 3: Computational grids.

\begin{tabular}{lcccc}
\hline \multirow{2}{*}{ Propeller } & Nodes at rotating & \multicolumn{2}{c}{ Nodes at fixed } & \multirow{2}{*}{ Total nodes } \\
& Hexa & Hexa & Hybrid & \\
\hline E779A & 644655 & & 163041 & 807696 \\
PPTC & 1838655 & 275680 & & 2114335 \\
\hline
\end{tabular}

As far as the discretization of the Fixed region is concerned, in the case of the E779A propeller a hybridunstructured mesh was used, while in the case of the PPTC propeller a hexa-structured mesh was employed. In the case of E779A propeller, we preferred to use a hybridunstructured mesh for Fixed, because we observed that using the hexastructured approach the block decomposition led to highly distorted elements in the twisted region over Rotating.

It is important to clarify that both mesh arrangements used here proved to guarantee mesh independent results in former studies $[33,34]$, and the average value of $y^{+}$measured on blade surfaces was approximatively equal to 38 for E779A propeller and 32 for PPTC propeller. Figure 10 illustrates the blade surface meshes of both propellers.

4.4. Results and Discussion. Before discussing the results, it is important to point out that in all the simulations the maximum density ratio was limited to $\rho_{L} / \rho_{V}=1000$ in order to guarantee solver stability. Moreover, in the case of the Kunz model the value of $t_{\infty}$ was set according to the operational conditions as $t_{\infty}=C_{0.7 R} / \sqrt{U^{2}+(2 \pi n 0.7 R)^{2}}$, where $C_{0.7 R}$ was the propeller blade chord at $70 \%$ of propeller radius $R(\mathrm{~m})$ and $U(\mathrm{~m} / \mathrm{s})$ the incoming free-stream velocity.

The numerical results were quantitatively compared considering the global values of the problem represented by the thrust $K_{T}$ and torque $K_{Q}$ coefficients defined as follows:

$$
\begin{aligned}
& K_{T}=\frac{T}{\rho_{L} n^{2} D^{4}}, \\
& K_{Q}=\frac{Q}{\rho_{L} n^{2} D^{5}},
\end{aligned}
$$

where $T(\mathrm{~N})$ and $Q(\mathrm{Nm})$ were the propeller thrust and torque, $n$ (rps) was the propeller rotational speed and $D(\mathrm{~m})$ the propeller diameter. 


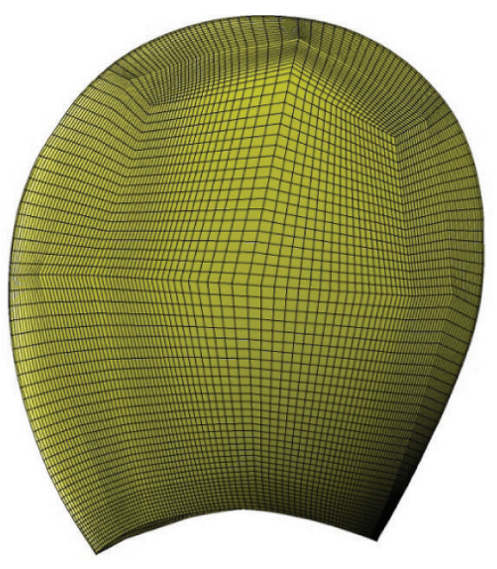

(a)

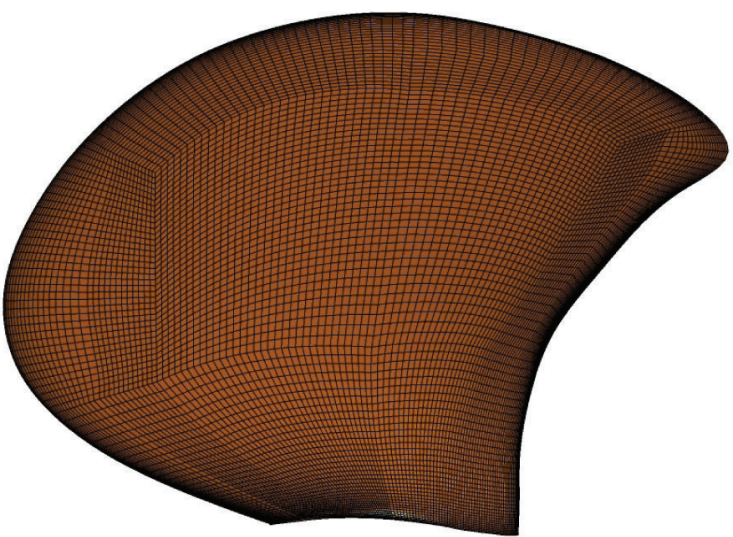

(b)

FIGURE 10: Suction side surface meshes, E779A propeller (a), PPTC propeller (b).

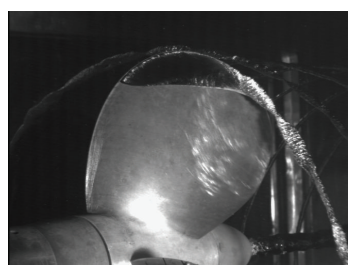

Exp. $J=0.71, \sigma_{n}=1.763$

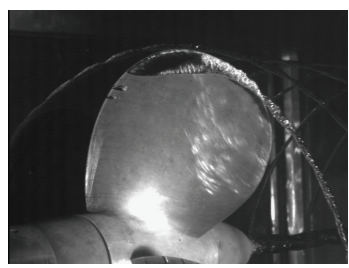

Exp. $J=0.77, \sigma_{n}=1.783$

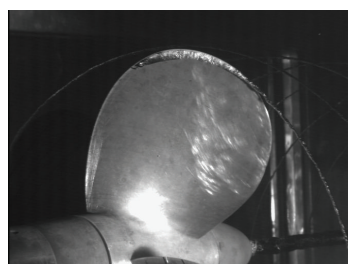

Exp. $J=0.83, \sigma_{n}=2.063$

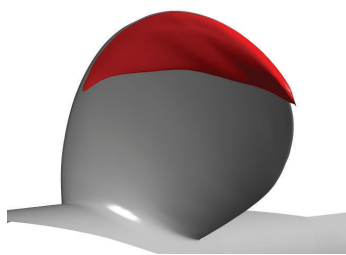

Zwart

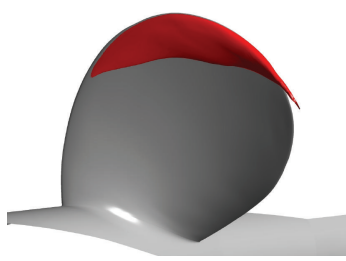

Zwart

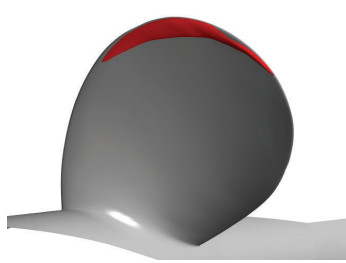

Zwart

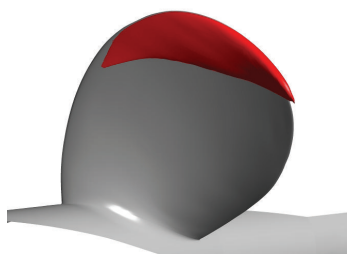

FCM

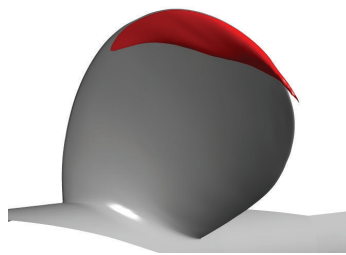

FCM

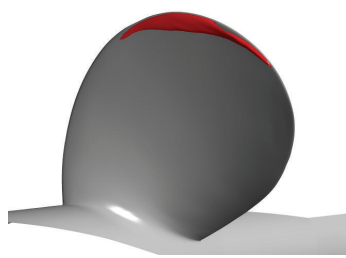

FCM

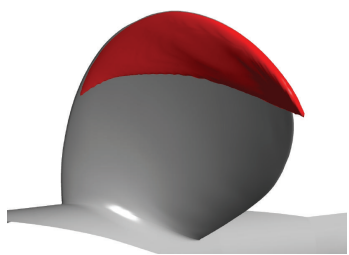

Kunz

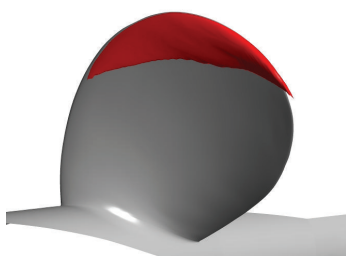

Kunz

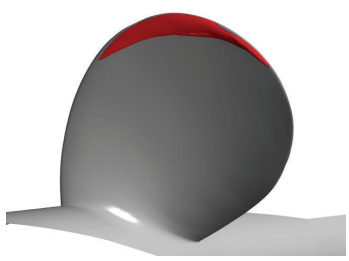

Kunz

FIGURE 11: Comparison of the cavitation patterns for three different operational conditions for the E779A propeller. The numerical cavitation patterns, obtained using the three different mass transfer models, are depicted as isosurfaces of vapour volume fraction $\alpha=0.5$.

The relative percentage errors of the computed $K_{T}, K_{Q}$ values were defined as follows:

$$
\begin{aligned}
& \Delta K_{T}(\%)=\frac{K_{T, \mathrm{CFD}}-K_{T, \mathrm{EXP}}}{K_{T, \mathrm{EXP}}} \cdot 100, \\
& \Delta K_{Q}(\%)=\frac{K_{Q, \mathrm{CFD}}-K_{Q, \mathrm{EXP}}}{K_{Q, \mathrm{EXP}}} \cdot 100,
\end{aligned}
$$

where $K_{T, \mathrm{CFD}}, K_{Q, \mathrm{CFD}}$ were the numerical values and $K_{T, \mathrm{EXP}}$, $K_{Q, \mathrm{EXP}}$, the experimental values.
For a qualitative comparison, also sketches of cavitation patterns were considered.

Next, the results obtained for the E779A propeller are presented first, followed by those obtained for the PPTC propeller.

4.4.1. E779A Propeller. The cavitating flow predictions were carried out following the experimental setup suggested by INSEAN [35] and reported in [6, 23].

In Figure 11, the cavitation patterns, predicted for three particular operational conditions and using the three 


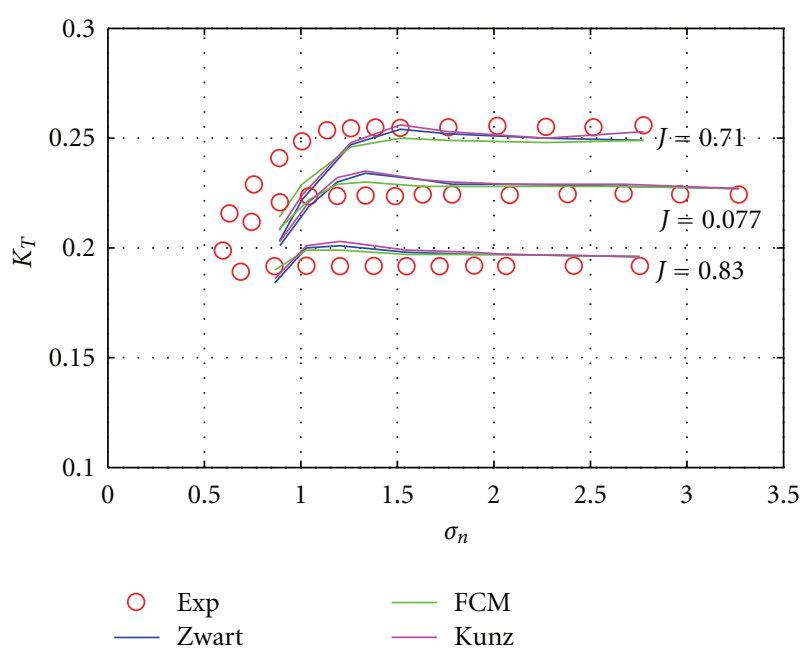

Figure 12: Influence of the cavitation number on the thrust coefficient for the E779A propeller.

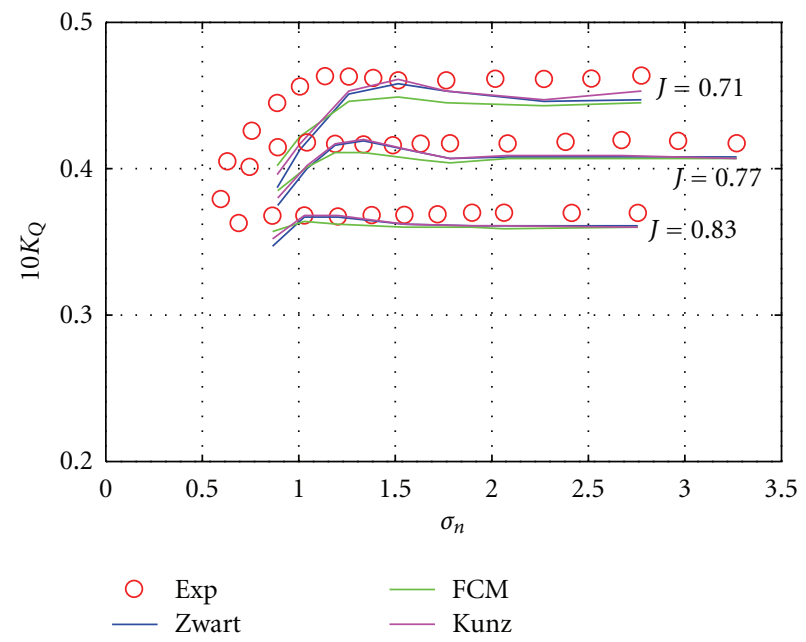

FIGURE 13: Influence of the cavitation number on the thrust coefficient for the E779A propeller.

different mass transfer models, are qualitatively compared. From Figure 11, it is possible to note that the cavitation patterns predicted with the three different mass transfer models were very similar to each other. Even though the cavitation patterns were slightly overestimated, from Table 4 it is possible to appreciate that for these operational conditions the predicted values of thrust and torque coefficients compared very well with the experimental data.

Figures 12 and 13 show that also covering a wider range of operational conditions, the results obtained using the different mass transfer models were similar. However, the effect of cavitation number on thrust and torque was not properly reproduced leading to the premature prediction of the thrust breakdown.

This discrepancy between the numerical results and experimental measurements could be probably related to the overestimation of the cavitation pattern (see Figure 11).
TABLE 4: Relative percentage errors for the E779A propeller.

\begin{tabular}{lccccccc}
\hline & \multicolumn{3}{c}{$\Delta K_{T}(\%)$} & \multicolumn{3}{c}{$\Delta K_{Q}(\%)$} \\
& $\sigma_{n}$ & Zwart & FCM & Kunz & Zwart & FCM & Kunz \\
\hline 0.71 & 1.763 & -1.01 & -2.27 & -0.61 & -1.63 & -3.07 & -1.60 \\
0.77 & 1.783 & 2.11 & 1.46 & 2.35 & -2.59 & -3.18 & -2.52 \\
0.83 & 2.063 & 2.77 & 2.51 & 2.85 & -2.33 & -2.52 & -2.35 \\
\hline
\end{tabular}

4.4.2. PPTC Propeller. The numerical predictions were carried out following the instruction kindly provided by the smp'11 workshop organizers and thus the cavitating flow predictions were carried out according to the thrust identity ( $K_{T}$ noncavitating flow conditions) for $J=1.019, J=$ 1.269 , and $J=1.408$. In our case, in order to fulfil this requisite within an error tolerance of $3 \%$ in the case of the smaller $J$ value, that is, $J=1.019$, the inflow velocity had to be slightly reduced leading to $J=1.016$. Table 5 collects the $K_{T}$ values predicted for the three different operational conditions and those obtained experimentally. Figure 14 shows that the cavitation patterns obtained using the three different calibrated mass transfer models were, in general, very similar to each other and compared very well with the experimental visualizations for the considered operational conditions, that is, $\left(J=1.019, \sigma_{n}=2.024\right),(J=1.269$, $\left.\sigma_{n}=1.424\right)$, and $\left(J=1.408, \sigma_{n}=2.000\right)$. From Table 6 , it is possible to note that the values of the thrust and torque coefficients predicted using alternatively the three different calibrated mass transfer models were surprisingly very close to each other and in excellent agreement with the experimental data for $J=1.019, \sigma_{n}=2.024$, even though the shape of the cavitation pattern was properly reproduced only with the FCM. Regarding other operational conditions, the differences between the predicted and experimental values were slightly more pronounced for the Zwart model at $(J=$ $\left.1.269, \sigma_{n}=1.424\right)$ and for the FCM model at $(J=1.408$, $\left.\sigma_{n}=2.000\right)$.

\section{Concluding Remarks}

In this study, a well-known commercial CFD solver, that is, ANSYS-CFX 12, was used to predict the cavitating flow around model scale propellers working in uniform inflow. The simulations were carried out using the homogeneous model, and the influence of three widespread mass transfer models, on the accuracy of the numerical predictions, was evaluated. The considered mass transfer models share the common feature of employing empirical coefficients to adjust the mass transfer rate due to the evaporation and condensation processes, which can affect both accuracy and stability of the numerical predictions. Thus, for a fair and congruent comparison the empirical coefficients of the three mass transfer models were first properly calibrated using an optimization strategy driven by the modeFRONTIER 4.2 optimization framework. The models were calibrated on the basis of the two-dimensional stable sheet cavity flow around a hydrofoil. 


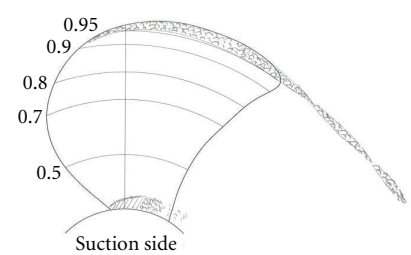

Exp. $J=1.019, \sigma_{n}=2.024$

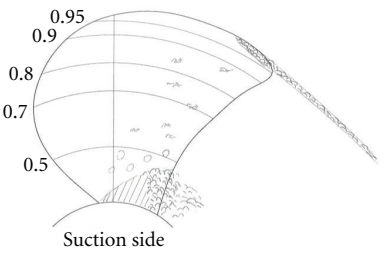

Exp. $J=1.269, \sigma_{n}=1.424$

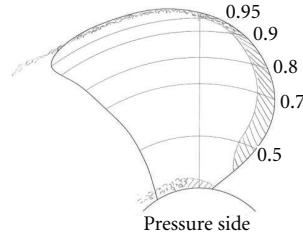

Exp. $J=1.408, \sigma_{n}=2$

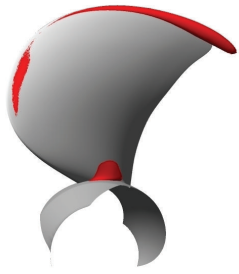

Zwart

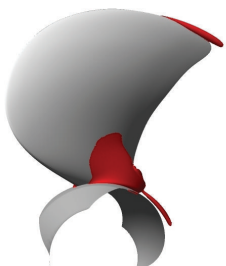

Zwart

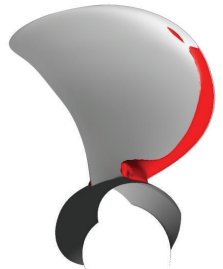

Zwart

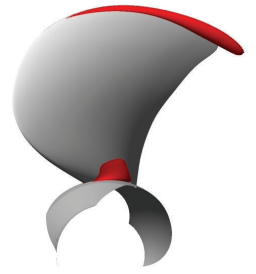

FCM

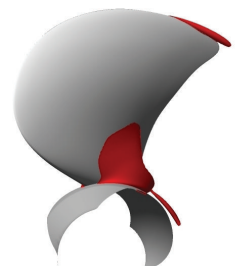

FCM

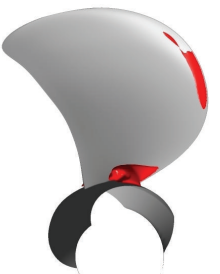

FCM

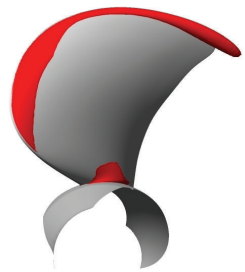

Kunz

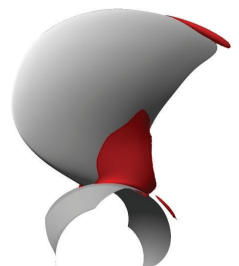

Kunz

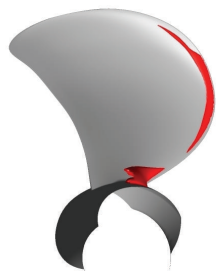

Kunz

FIGURE 14: Comparison of the cavitation patterns for three different operational conditions for the PPTC propeller. The numerical cavitation patterns, obtained using the three different mass transfer models, are depicted as isosurfaces of vapour volume fraction $\alpha=0.5$.

TABle 5: Fulfil of thrust identity ( $K_{T}$ non-cavitating) for PPTC propeller.

\begin{tabular}{lcccc}
\hline & CFD & & \multicolumn{2}{c}{ Exp. } \\
$J$ & $K_{T}$ & $J$ & $K_{T}$ \\
\hline 1.016 & 0.382 & 1.019 & 0.387 \\
1.269 & 0.240 & 1.269 & 0.245 \\
1.408 & 0.166 & 1.408 & 0.167 \\
\hline
\end{tabular}

TABLE 6: Relative percentage errors for the PPTC propeller.

\begin{tabular}{lccccccc}
\hline & \multicolumn{4}{c}{$\Delta K_{T}(\%)$} & \multicolumn{3}{c}{$\Delta K_{Q}(\%)$} \\
& $\sigma_{n}$ & Zwart & FCM & Kunz & Zwart & FCM & Kunz \\
\hline 1.019 & 2.024 & 0.13 & 0.40 & 0.67 & -1.63 & -1.11 & -1.22 \\
1.269 & 1.424 & -5.04 & -1.65 & 1.74 & -4.15 & -0.82 & 3.45 \\
1.408 & 2.000 & -2.35 & -4.55 & -2.35 & -1.84 & -2.45 & -2.45 \\
\hline
\end{tabular}

The calibrated models were then used to predict the cavitating flow around the model scale propellers E779A and PPTC.

No significant differences were observed in the simulations performed using alternatively the three different calibrated mass transfer models. For selected operational conditions, corresponding to the appearance of partial and tip-vortex cavitation, the numerical results compared well with the experimental data, even though the tendency to slightly overestimate the cavity extension was observed. Unfortunately, considering other more severe operational conditions, the numerical results showed significant discrepancies with the experimental data. Further investigations on this aspect are in progress.

\section{Acknowledgments}

This study was performed in the context of the project OpenSHIP, supported by Regione FVG_POR FESR 20072013 Obiettivo competitività regionale e occupazione. The authors wish to thank INSEAN, and in particular Dr. Francesco Salvatore, for the assistance and for providing the geometry and the experimental measurements for the E779A propeller.

\section{References}

[1] P. J. Zwart, A.G. Gerber, and T. Belamri, "A two-phase model for predicting cavitation dynamics," in Proceedings of the International Conference on Multiphase Flow (ICMF '04), Yokohama, Japan, 2004.

[2] S. Frikha, O. Coutier-Delgosha, and J. A. Astolfi, "Influence of the cavitation model on the simulation of cloud cavitation on 2D foil section," International Journal of Rotating Machinery, vol. 2008, Article ID 146234, 12 pages, 2008. 
[3] R. F. Kunz, D. A. Boger, D. R. Stinebring et al., "A preconditioned Navier-Stokes method for two-phase flows with application to cavitation prediction," Computers and Fluids, vol. 29, no. 8, pp. 849-875, 2000.

[4] A. K. Singhal, M. M. Athavale, H. Li, and Y. Jiang, "Mathematical basis and validation of the full cavitation model," Journal of Fluids Engineering, vol. 124, no. 3, pp. 617-624, 2002.

[5] M. Morgut, E. Nobile, and I. Biluš, "Comparison of mass transfer models for the numerical prediction of sheet cavitation around a hydrofoil," International Journal of Multiphase Flow, vol. 37, no. 6, pp. 620-626, 2011.

[6] F. Pereira, F. Salvatore, and F. D. Felice, "Measurement and modeling of propeller cavitation in uniform inflow," Journal of Fluids Engineering, vol. 126, no. 4, pp. 671-679, 2004.

[7] H. J. Heinke, "Potsdam propeller test case (PPTC), cavitation tests with the model propeller," Tech. Rep. VP1304, SVA (Potsdam Model Basin), 2011.

[8] A. H. Koop, Numerical simulation of unsteady three-dimensional sheet cavitation [Ph.D. thesis], University of Twente, 2008.

[9] Y. A. Bouziad, Phisical modelling of leading edge cavitation: computational methodologies and application to hydraulic machinery [Ph.D. thesis], Ecole Polytechnique Federale de Lausanne, 2005.

[10] ANSYS, ANSYS CFX-Solver Theory Guide, Release 12.0, 2009.

[11] H. K. Versteeg and W. Malalasekera, An Introduction to Computational Fluid Dynamics, the Finite Volume Method, Pearson Education Limited, 2nd edition, 2007.

[12] J. Blazek, Computational Fluid Dynamics: Principles and Applications, Elsevier, New York, NY, USA, 2nd edition, 2005.

[13] C. E. Brennen, Fundamentals of Multiphase Flows, Cambridge University Press, New York, NY, USA, 2005.

[14] http://www.ansys.com.

[15] http://www.simerics.com.

[16] T. Huuva, Large eddy simulation of cavitating and noncavitating flow [Ph.D. thesis], Department of Shipping and Marine Technology, Chalmers University of Technology, Gothenburg, Sweden, 2008.

[17] C. L. Merkle, J. Feng, and P. E. O. Buelow, "Computational modeling of the dynamics of sheet cavitation," in Proceedings of the 3rd International Symposium on Cavitation, vol. 2, Grenoble, France, 1998.

[18] http://www.openfoam.com.

[19] ESTECO, modeFRONTIER 4 User Manual, 2009.

[20] A. Clarich, G. Mosetti, V. Pediroda, and C. Poloni, "Application of evolutionary algorithms and statistical analysis in the numerical optimization of an axial compressor," International Journal of Rotating Machinery, vol. 2, pp. 143-151, 2005.

[21] Y. T. Shen and P E. Dimotakis, "The influence of surface cavitation on hydrodynamic forces," in Proceedings of the 22nd American Towing Tank Conference (ATTC'89), pp. 44-453, St. Johns, Canada, 1989.

[22] S. S. Rao, Engineering Optimization-Theory and Practice, John Wiley \& Sons, New York, NY, USA, 1996.

[23] F. Salvatore H. Streckwall and T. van Terwisga, "Propeller cavitation modelling by CFD-results from the VIRTUE 2008," in Proceedings of the 1st International Symposium on Cavitation (SMP '09), pp. 362-371, Trondheim, Norway, June 2009.

[24] G. N. V. B. Vaz, Modelling of sheet cavitation on hydrofoils and marine propellers using boundary element methods [Ph.D. thesis], Universidade Tecnica de Lisboa, 2005.
[25] R. E. Bensow and G. Bark, "Implicit LES predictions of the cavitating flow on a propeller," Journal of Fluids Engineering, vol. 132, no. 4, Article ID 041302, 2010.

[26] http://www.marinepropulsors.com.

[27] http://www.sva-potsdam.de/pptc.

[28] U. H. Barkmann, "Potsdam propeller test case (PPTC), open water tests with the model propeller," Tech. Rep. VP1304, SVA (Potsdam Model Basin), 2011.

[29] K. P. Mach, "Potsdam propeller test case (PPTC), LDV velocity measurements with the model propeller," Tech. Rep. VP1304, SVA (Potsdam Model Basin), 2011.

[30] F. R. Menter, "Two-equation eddy-viscosity turbulence models for engineering applications," AIAA Journal, vol. 32, no. 8, pp. 1598-1605, 1994.

[31] N. Berchiche and C.E. Janson, "Grid influence on the propeller open-water performance and flow field," Ship Technology Research, vol. 55, pp. 87-96, 2008.

[32] M. Abdel-Maksoud, F. Menter, and H. Wuttke, "Viscous flow simulations for conventional and high-skew marine propellers," Ship Technology Research, vol. 45, no. 2, pp. 64-71, 1998.

[33] M. Morgut and E. Nobile, "Influence of grid type and turbulence model on the numerical prediction of the flow around marine propellers working in uniform inflow," Ocean Engineering, vol. 42, pp. 26-34, 2012.

[34] M. Morgut and E. Nobile, "Numerical predictions of the cavitating and non-cavitating flow around the model scale propeller pptc," in Proceedings of the Workshop on Cavitation and Propeller Performance, the 2nd International Symposium on Marine Propulsors (SMP '11), Hamburg, Germany, 2011.

[35] F. Salvatore, Private communication, 2010. 

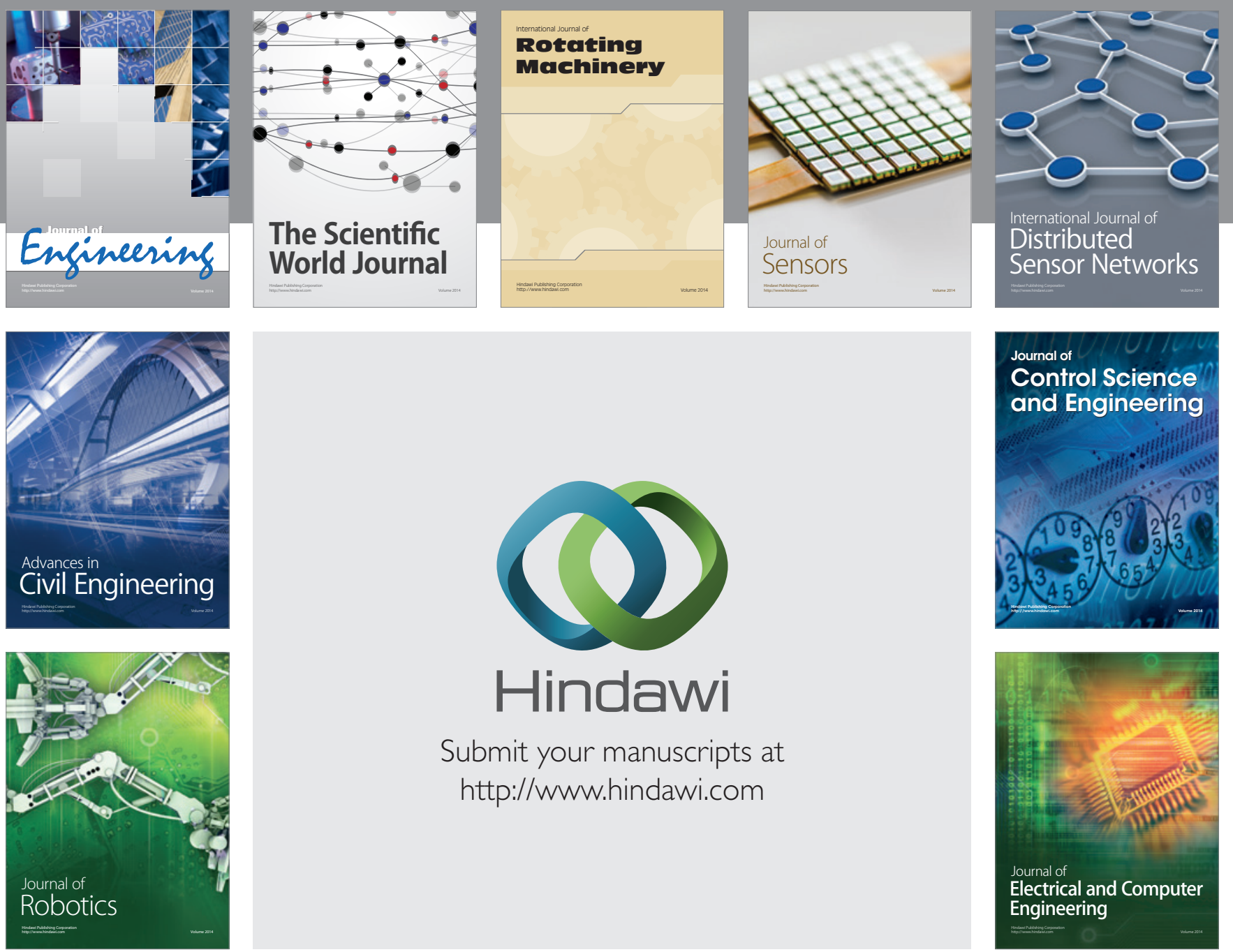

Submit your manuscripts at

http://www.hindawi.com
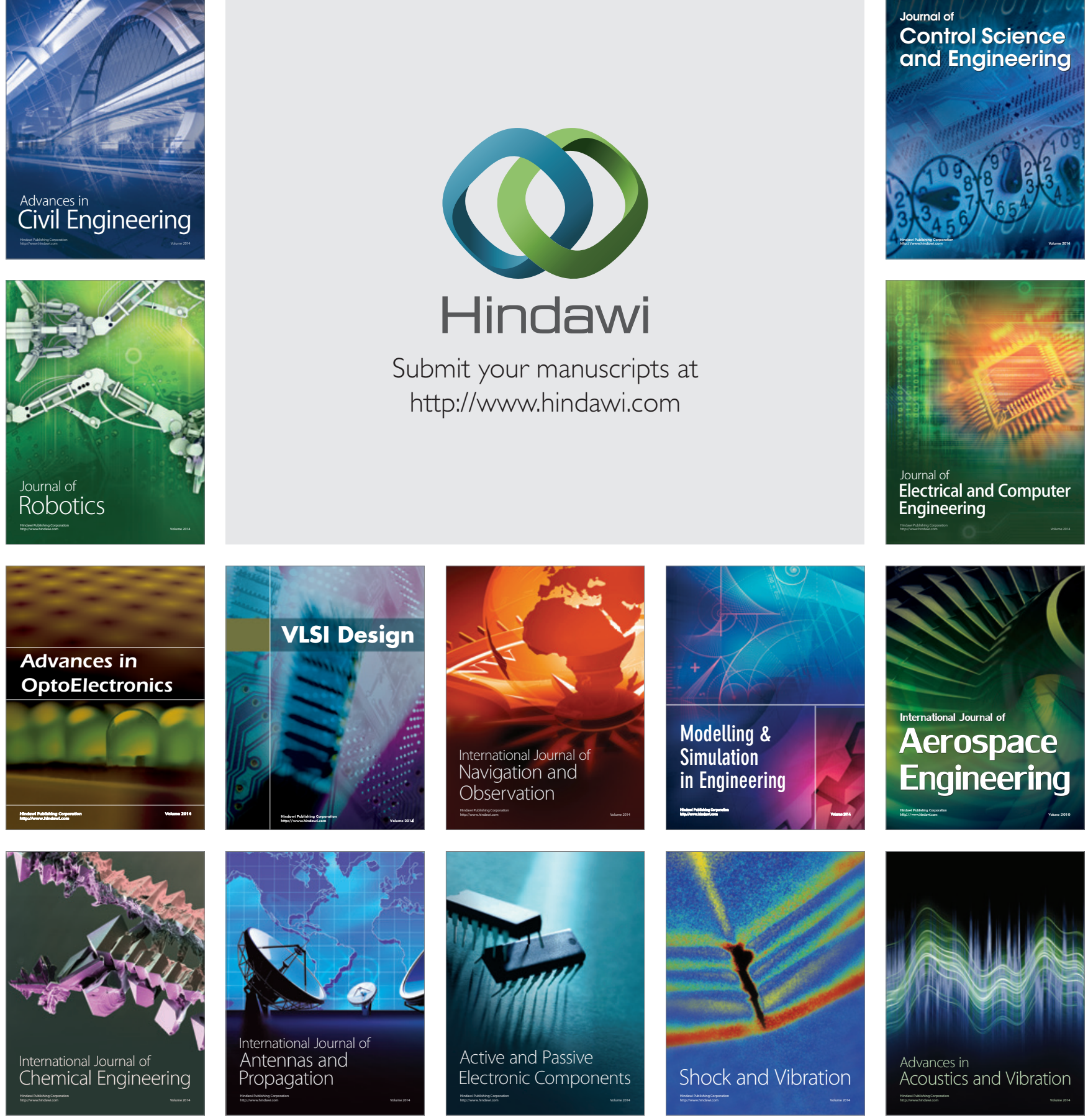